\title{
Effects of dissipation rate and diffusion rate of \\ the progress variable on local fuel burning rate in premixed turbulent flames
}

\author{
Bruno Savard ${ }^{a, b, 1}$ and Guillaume Blanquart ${ }^{c}$ \\ ${ }^{a}$ Graduate Aerospace Laboratories, California Institute of Technology, Pasadena, \\ CA 91125, USA \\ ${ }^{b}$ Institute of Heat Engineering, Warsaw University of Technology, 00-665 Warsaw, \\ Poland \\ ${ }^{c}$ Mechanical Engineering Department, California Institute of Technology, \\ Pasadena, CA 91125, USA
}

\begin{abstract}
The validity of the premixed flamelet equations and the dependence of the fuel burning rate on the parameters involved in these equations have been investigated using a large series of direct numerical simulations of turbulent premixed flames in the thin reaction zones (TRZ) and the distributed reaction zones (DRZ) regimes. Methane, toluene, $n$-heptane, and iso-octane fuels were considered over a wide range of unburnt conditions and turbulence characteristics. Flames with unity and nonunity Lewis numbers were investigated separately to isolate turbulence-chemistry interaction from differential diffusion effects. In both cases, the flamelet equations, which rely on the assumption of a thin reaction zone, are locally valid throughout the TRZ regime, more precisely up to a Karlovitz number at the reaction zone of 10 (based on the definition used in this paper). Consistent with this result, in the unity
\end{abstract}


Lewis number limit, the fuel burning rate is strongly correlated with the dissipation rate of the progress variable, the only parameter in the flamelet equations. In the non-unity Lewis number case, the burning rate is a strong function of both the dissipation rate and the diffusion rate, both of which are parameters in the flamelet equations. In particular, the correlation with these parameters is significantly better than with curvature or tangential strain rate.

Key words: turbulent premixed flame, reaction zone, flamelet, dissipation rate, hydrocarbon fuels.

\section{Introduction}

Turbulent premixed flames play a major role in modern internal combustion engines and gas-turbine combustors. These premixed flames often involve large hydrocarbon fuels and typically fall in the thin reaction zones (TRZ) regime $[1-5]$. To promote an efficient engine design process, a better understanding of turbulent premixed flames in this regime, and with such fuels, is desirable.

In previous studies $[6,7]$, we investigated, with direct numerical simulations (DNS), $n_{-} \mathrm{C}_{7} \mathrm{H}_{16} /$ air premixed turbulent flames at high Karlovitz number (TRZ regime), with and without differential diffusion. The following results were identified:

$\overline{1}$ Corresponding author

Email address: b.savard@unsw.edu.au

Present address: School of Mechanical and Manufacturing Engineering, University of New South Wales, Kensington, NSW 2052, Australia 
(1) largely thickened preheat zone

(2) thin reaction zone

(3) large fluctuations around $<\dot{\omega}_{F} \mid c>$

(4) for unity Lewis numbers:

$$
<\dot{\omega}_{F} \mid c>\approx \dot{\omega}_{F, \operatorname{lam}}(c),
$$

(5) for non-unity Lewis numbers:

$$
<\dot{\omega}_{F} \mid c>\not \approx \dot{\omega}_{F, \text { lam }}(c),
$$

where $\dot{\omega}_{F}$ is the fuel burning rate, and $c$ is a progress variable. $\langle\cdot \mid \psi\rangle$ denotes the mean conditional to the variable $\psi$. Lapointe $e t$ al. recently obtained similar results for $n$-heptane/air (over a wider range of Karlovitz numbers) [8,9], iso-octane/air [9], and toluene/air [9] flames with both high and low unburnt temperatures. Consistent results were also obtained for iso-octane/air flames in the TRZ regime over a wide range of pressures [10]. (While similar results are expected for all heavy hydrocarbon fuels, extension to certain light fuels such as hydrogen may not be warranted.)

It is important to note that broadened preheat zone (point 1), and yet thin reaction zone (point 2), have been observed beyond the traditional boundaries of the TRZ regime (i.e. at Karlovitz numbers higher than theoretically predicted [11]) [12]. Burning rate fluctuations (point 3) have also been identified in various other DNS studies [13-16]. Such source term fluctuations in turbulent flames have commonly been attributed to stretching effects, i.e. effects of curvature and strain rate $[13,17,16,18,19]$. However, these results were obtained at relatively low Karlovitz numbers, namely in the wrinkled/corrugated flamelet 
regimes and at the lower limit of the TRZ regime. In contrast, no strong correlation was identified between burning rate and strain rate or curvature at larger Karlovitz numbers [7].

The effect of the dissipation rate

$$
\chi=2 \alpha|\nabla c|^{2},
$$

with $\alpha$ the thermal diffusivity, on the burning rate in premixed turbulent flames has also been previously discussed in the literature. For instance, Bray [20], under BML assumptions, showed that the mean (Reynolds-averaged) source term should be proportional to the mean dissipation rate. Lipatnikov et al. [21] recently showed, using DNS data, that this result is valid in the wrinkled/corrugated flamelet regimes. Bray's model has later been improved for application to both Reynolds-averaged Navier-Stokes simulations (RANS) and large eddy simulations (LES) (e.g. Ref. [22-26]). In conditional mean closure (CMC) methods, the scalar dissipation rate is an essential term that needs closure [27,28]. Veynante and Vervisch [29] also discuss the importance of the scalar dissipation rate for turbulent combustion modeling. Nevertheless, none of these models uses the dissipation rate to capture the burning rate fluctuations (only the mean or filtered burning rate).

In the absence of differential diffusion, Kolla [30] showed that, if the species mass fractions are only a function of the progress variable, then

$$
\dot{\omega}_{c} \frac{\partial Y_{i}}{\partial c}=\frac{\rho \chi}{2} \frac{\partial^{2} Y_{i}}{\partial c^{2}}+\dot{\omega}_{i}
$$

where $Y_{i}$ is the $i$ th species mass fraction, $\rho$ is the density, and $\dot{\omega}_{i}$ is the chemical source term of species $i$. In analogy with non-premixed flamelet equations, this constitutes a premixed flamelet equation. Nguyen et al. [31] also derived 
a similar equation and showed the importance of the progress variable dissipation rate. Recently, Lapointe and Blanquart [9] investigated a wide range of turbulent premixed flames in the TRZ and the distributed reaction zones (DRZ) regimes. They showed that the burning rate fluctuations are strongly correlated with the dissipation rate on the iso-temperature corresponding to the peak burning rate. It is important to highlight that these results were obtained in the absence of differential diffusion.

The inclusion of differential diffusion effects in non-premixed flamelet equations has been the subject of multiple studies [32-35] starting with Pitsch and Peters [36]. By considering a progress variable instead of a mixture fraction and using a coordinate transformation similar to Xuan et al. [34], we derived 3D premixed flamelet equations with non unity Lewis numbers in Ref. [37]. In analogy to the non-unity Lewis number non-premixed case, an additional parameter was identified in the premixed flamelet equations, namely the diffusion rate of the progress variable, $\nabla \cdot(\rho \alpha \nabla c)$. To the best of the authors' knowledge, the dependence of burning rate fluctuations on this quantity in turbulent premixed flames has never been investigated.

In light of the above discussion, the first objective is to test the validity of the premixed flamelet equations in turbulent premixed flames across the TRZ regime up to the DRZ regime over a wide range of unburnt conditions and mixtures (with particular attention to large hydrocarbon fuels). Both unity and non-unity Lewis number cases will be considered. A value for the largest Karlovitz number at the reaction zone for which the equations are valid can then be provided as a by-product. Note that the validity of flamelet equations in turbulent flames was recently tested by Scholtissek et al. [35] but only for non-premixed flames. The second objective is to assess if burning rate 
fluctuations can be locally attributed to variations in the parameters from the flamelet equations. It is beyond the scope of this work to provide a closed model to be implemented in future LES. The focus is placed entirely on providing physical and mathematical explanations to these burning rate fluctuations.

The paper is organized as follows. The non-unity Lewis number premixed flamelet equations are reviewed in Section 2. An overview of the DNS data set on which the analysis is performed is presented in Section 3. For the analysis, the unity Lewis number limit is considered first in Section 4. The analysis is then repeated in Section 5 for the non-unity Lewis number cases and differential diffusion effects are highlighted. Finally, a summary and conclusions are provided in Section 6 .

\section{One-dimensional flamelet equations}

The one-dimensional flamelet equations presented in this section, which were first introduced in Ref. [37], are the premixed equivalent of the non-premixed flamelet equations derived in Xuan et al. [34]. For complete details of the derivation, the reader is referred to [37] (Chapter 7).

The premixed flamelet equations result from the following coordinate transformation applied to the species transport and temperature equations:

$$
\begin{aligned}
& \left(x_{1}, x_{2}, x_{3}, t\right) \\
& \rightarrow\left(c\left(x_{1}, x_{2}, x_{3}, t\right), c_{2}\left(x_{1}, x_{2}, x_{3}, t\right),\right. \\
& \left.\quad c_{3}\left(x_{1}, x_{2}, x_{3}, t\right), \tau\right),
\end{aligned}
$$


with

$$
\nabla c \cdot \nabla c_{2}=0, \quad \text { and } \quad \nabla c \cdot \nabla c_{3}=0
$$

i.e. the variables $c_{2}$ and $c_{3}$ lie in the surface of constant $c$. Note that these variables can be curvilinear. The progress variable $c$ is considered to be a linear combination of species mass fractions $Y_{j}$ in the derivation, i.e. $c=\sum_{j=1}^{N} b_{j} Y_{j}$, where the $b_{j}$ 's are arbitrary coefficients and $N$ is the number of species. For the results shown in Sections 4 and 5, $c=Y_{\mathrm{H}_{2}}+Y_{\mathrm{H}_{2} \mathrm{O}}+Y_{\mathrm{CO}}+Y_{\mathrm{CO}_{2}}$. (Note that we did not normalize the progress variable to unity. Hence, it ranges from 0 to 0.25 for the present $n-\mathrm{C}_{7} \mathrm{H}_{16}$ /air flames.)

The next step consists of assuming that, in the vicinity of the reaction zone, all tangential derivatives $\left(\partial / \partial c_{k}\right)$ are negligible compared to the normal derivatives $(\partial / \partial c)$. It is further assumed that the dependence on $\tau$ in the transformed coordinate system is negligible. Note that the coordinate $c$ is still a function of time $t$. These assumptions are expected to be more valid at lower Karlovitz number (thin reaction zones) than at high Karlovitz number (distributed reaction zones). This will be assessed in Sections 4 and 5. These two assumptions will be used (and tested) in the rest of this paper and are therefore listed here for clarity:

$$
\begin{aligned}
& \partial / \partial c_{k} \ll \partial / \partial c, \text { for } k=2,3, \\
& \partial / \partial \tau=0 .
\end{aligned}
$$

With these assumptions (note that no assumption is made on the species Lewis 
numbers), the flamelet equations for the mass fractions $Y_{i}$ read

$$
\begin{aligned}
& \underbrace{\dot{\omega}_{c} \frac{\mathrm{d} Y_{i}}{\mathrm{~d} c}}_{\text {convection }} \\
& =\underbrace{\frac{\rho \chi}{2 L e_{i}} \frac{\mathrm{d}^{2} Y_{i}}{\mathrm{~d} c^{2}}}_{\text {differential diffusion-induced convection }}+\underbrace{\dot{\omega}_{i}}_{\text {diffusion }} \\
& +\underbrace{\left[\xi\left(\frac{1}{L e_{i}}-\frac{1}{L e_{c}}\right)+\frac{\rho \chi}{2} \frac{\mathrm{d}}{\mathrm{d} c}\left(\frac{1}{L e_{i}}-\frac{1}{L e_{c}}\right)\right] \frac{\mathrm{d} Y_{i}}{\mathrm{~d} c}}_{\text {chemical source }} \\
& +\underbrace{\nabla \cdot\left(\rho Y_{i} \mathbf{V}_{\mathbf{c}, \mathbf{i}}\right)-\sum_{j=1}^{N} b_{j} \nabla \cdot\left(\rho Y_{j} \mathbf{V}_{\mathbf{c}, \mathbf{j}}\right) \frac{\mathrm{d} Y_{i}}{\mathrm{~d} c},}_{j=1}
\end{aligned}
$$

where

$$
\xi=\nabla \cdot(\rho \alpha \nabla c)
$$

is the diffusion rate of the progress variable. The flamelet equation for temperature can be found in Ref. [37] (Eq. 7.24) for completeness. The term $\nabla \cdot\left(\rho Y_{i} \mathbf{V}_{\mathbf{c}, \mathbf{i}}\right)$ is expanded in Ref. [37] (Eq. 7.23). In the above equations, $L e_{i}$ is the Lewis number of species $i$. The correction velocity $\mathbf{V}_{\mathbf{c}, \mathbf{i}}$ accounts for gradients in the mixture molecular weight as well as ensures zero net diffusion flux. Note that the inverse of the Lewis number of the progress variable can be expressed as $\frac{1}{L e_{c}}=\sum_{j=1}^{N} \frac{b_{j}}{L e_{j}} \frac{\mathrm{d} Y_{j}}{\mathrm{~d} c}$ (more details Ref. [37], Section 7.1).

It is important to note that the terms labeled "convection" or "diffusion" are consistent with the nomenclature used in Xuan et al. [34] and refer to terms involving first and second derivatives of species mass fractions, respectively.

These equations form a system of ordinary differential equations in which the $Y_{i}$ are the unknowns, $c$ is the variable, and the dissipation rate $\chi(c)$ and the diffusion rate $\xi(c)$ are the parameters. Given the two assumptions made in this section, each of the terms in the above equations are only a function of $c, \chi$ 
and $\xi$. Note that, following the detailed derivation in Ref. [34], the parameter $\xi(c)$ includes curvature effects and may be expressed as

$$
\xi=\frac{1}{4}\left[\frac{\mathrm{d}}{\mathrm{d} c}(\rho \chi)+\frac{\chi}{\alpha} \frac{\mathrm{d}}{\mathrm{d} c}(\rho \alpha)\right]-\rho \kappa\left(\frac{\chi \alpha}{2}\right)^{1 / 2},
$$

where $\kappa=\nabla \cdot(-\nabla c /|\nabla c|)$ is the mean curvature of the progress variable iso-surfaces.

The main difference between these premixed flamelet equations and the nonpremixed flamelet equations derived by Xuan et al. [34] is the presence of the convective term due to the source term of the progress variable (term on the left hand side). In Nguyen et al. [31], similar flamelet equations were obtained for the premixed case, with the exception that the velocity correction and the differential diffusion-induced convection terms were argued to be negligible. These terms were included in the equations derived by Lodier et al. [38]. The latter term is important in the present $n$-heptane turbulent flames (Section 5). It is important to note that the equations Nguyen et al. and Lodier et al. derived are different from those presented in this paper. The difference resides in Eq. 11; in their work, the operators are projected on the $c$-space and therefore the curvature term is absent (more details in the supplemental material). It is shown in Section 5 that the curvature term should not be neglected in the flames considered in the present work.

\section{DNS data set}

The analysis will be performed on a large series of DNS of premixed turbulent flames. Both statistically steady, planar flames [6] and a highly sheared slot-burner flame [39] are considered. The simulations were chosen to cover 
a wide range of Karlovitz numbers ranging from the lower limit of the TRZ regime to the DRZ regime, as illustrated in Fig. 1. Large hydrocarbon fuels, namely $n$-heptane, iso-octane, and toluene are considered as these are often included in transportation fuel surrogates [40-43]. These fuels are associated with large Lewis numbers. In contrast, a smaller hydrocarbon fuel, methane (important for natural gas surrogates), which has a close to unity Lewis number, is also considered. In addition, a wide range of unburnt temperatures and background pressures are considered (representative of engine conditions). Finally, simulations with unity Lewis numbers (for all species) were also chosen to perform the analysis in the absence of differential diffusion.

The simulation parameters are listed in Tables 1,2 , and 3. Cases $\mathrm{B}_{n \mathrm{C} 7}$ and $\mathrm{B}_{n \mathrm{C} 7,1}$ were first presented in Ref. [6] and further used in Ref. [7,8,37,44,9]. Cases $\mathrm{A}_{n \mathrm{C} 7}$ to $\mathrm{D}_{n \mathrm{C} 7,1}$ were introduced and used by Lapointe et al. $[8,9]$. Lapointe and Blanquart [9] later introduced cases $\mathrm{B}_{\mathrm{CH} 4}, \mathrm{~B}_{\mathrm{CH} 4,1}, \mathrm{~B}_{\mathrm{ACH} 3}$, and $\mathrm{B}_{\mathrm{ACH} 3,1}$. More recently, cases $\mathrm{A}_{i \mathrm{C} 8, \mathrm{P} 1 \mathrm{~T} 3}, \mathrm{~A}_{i \mathrm{C} 8, \mathrm{P} 20 \mathrm{~T} 3}, \mathrm{~A}_{i \mathrm{C} 8, \mathrm{P} 20 \mathrm{~T} 8}$, and $\mathrm{B}_{i \mathrm{C} 8}$ were presented in Ref. [10]. Cases $\mathrm{AAA}_{n \mathrm{C} 7}$ and $\mathrm{AA}_{n \mathrm{C} 7}$ have not been presented previously. They correspond to case $\mathrm{A}_{n} \mathrm{C} 7$ with lower velocity fluctuations. The only parameters modified to obtain these two simulations are the forcing coefficient and the mean inflow velocity (more details in Ref. [7]). Each of these flames are statistically steady and planar.

In order to assess the effect of the integral length scale on the present analysis, the following flames were also considered: 1) case $\mathrm{B}_{n \mathrm{C} 7,4 L}[45]$ (see Table 1) which corresponds to case $\mathrm{B}_{n \mathrm{C} 7}$ (same fuel, unburnt conditions, Lewis numbers, and Karlovitz number) but with an integral length scale four times larger and 2) cases $\mathrm{S}^{1 / 4}, \mathrm{~S}^{1 / 2}$, and $\mathrm{S}^{3 / 4}$ (see Table 3) which correspond to the highest-Ka slot-burner methane/air flame of Sankaran et al. [39] at three axial 
locations $(1 / 4,1 / 2$, and $3 / 4$ of the domain height, as described in Ref. [39]).

All the simulations were performed with reduced chemical mechanisms which include 17 to 74 species and 73 to 976 elementary reactions. Constant Lewis numbers were used in all the simulations.

The reader is referred to the references listed above for further details about the respective simulations.

[Table 1 about here.]

[Table 2 about here.]

[Table 3 about here.]

In Tables 1 and 2, the labels for the cases are organized by Karlovitz number at the reaction zone, $K a_{\delta}$. It was shown in Lapointe et al. $[8,9]$ that the two most important parameters that control global dynamics of these flames are the Karlovitz number at the reaction zone and the Lewis number of the fuel. Effects of unburnt temperature [8,9] and background pressure [10] on global flame quantities were found to be negligible at constant $K a_{\delta}$ and $L e_{F}$. It is important to recall that the reaction zone was found to remain thin in all flames except cases $\mathrm{C}$ and $\mathrm{D}$, in which slightly distributed reaction zones were identified $[6,8-10,39]$.

[Fig. 1 about here.]

Finally, each of the statistically steady and planar flames was run until statistically steady state was reached and data was collected over the next 30 to 50 integral turnover times. A total of more than $12 \mathrm{~TB}$ of data were processed on NERSC's Hopper (Oakland, CA, USA) and CI TASK's Tryton (Gdańsk, 
Poland) to perform the present analysis.

\section{Unity Lewis number limit}

As a first step, the unity Lewis number limit is considered. In the absence of differential diffusion, the effect of turbulence on chemistry can be isolated. The validity of the assumptions leading to the flamelet equations is illustrated and then these equations are used to provide a physical explanation to the fuel burning rate fluctuations in these turbulent flames.

\subsection{Unity Lewis number flamelet equations}

In the absence of differential diffusion (i.e. by setting all Lewis numbers to unity), Eq. 9 reduces to

$$
\underbrace{\dot{\omega}_{c} \frac{\mathrm{d} Y_{i}}{\mathrm{~d} c}}_{\text {convection }}=\underbrace{\frac{\rho \chi}{2} \frac{\mathrm{d}^{2} Y_{i}}{\mathrm{~d} c^{2}}}_{\text {diffusion }}+\underbrace{\dot{\omega}_{i}}_{\text {chemical source }} .
$$

For completeness, the flamelet equation for temperature can be found in Ref. [37] (Eq. 7.16). These equations were identified previously by Kolla [30] who extended the work of Bilger [46] to premixed flames and by Nguyen et al. [31] who projected the set of species transport and temperature equations on the progress variable-space (their unity Lewis number purely premixed case).

Again, these equations form a system of ordinary differential equations in which the $Y_{i}$ (and $T$ ) are the unknowns, $c$ is the variable, and the dissipation rate $\chi(c)$ is the only parameter. Given the two assumptions made in Section 2, 
each of the terms in the above equations are only a function of $c$ and $\chi$.

\subsection{Validity of the flamelet equations}

To assess the two assumptions (Eq. 7 and 8), each of the terms in Eq. 12 (for several species) are evaluated point-wise in the turbulent flame. Then, the conditional mean and the standard deviation of these terms are computed and the results are presented and compared to the unstretched laminar flame solution in Fig. 2 for case $\mathrm{B}_{n \mathrm{C} 7,1}$. The species flamelet equations for $n$ - $\mathrm{C}_{7} \mathrm{H}_{16}$ (a reactant), $\mathrm{CO}$ (an intermediate that contributes to the progress variable), and $\mathrm{H}_{2} \mathrm{O}$ (a product that contributes to the progress variable) are considered. Note that due to the large number of grid points covering the reaction zone and the large number of data files considered (spanning over 30 turnover times), the statistics are smooth and well converged.

First, in contrast with the one-dimensional transport equations in physical space, the terms in the flamelet equations are only large in the reaction zone $(0.10<c<0.22)$, i.e. where the chemical source terms are non-negligible. In the preheat zone $(c<0.10)$, the species mass fraction vs. progress variable profiles are linear, even in the turbulent flames [6]. Therefore, the diffusion term (first term on the right hand side of Eq. 12) is close to zero. Without a surprise, all production terms are also close to zero in the preheat zone.

[Fig. 2 about here.]

Second, the mean profile for each of the terms collapses almost perfectly on the laminar solution. This is consistent with the results obtained in [6-8] where it was found that the mean turbulent flame structure (in terms of species 
mass fractions and chemical source terms vs. temperature) shows virtually no departure from the laminar flame solution in the absence of differential diffusion.

Third, and most importantly, the residual terms are small (both in terms of mean and rms) in comparison to the other terms. These residual terms (Eq. A.13 in Appendix A) correspond to the local balance of Eq. 12 and are indicative of the error introduced with the flamelet assumptions (Eq. 7 and 8). This result indicates that these two assumptions are valid for case $\mathrm{B}_{n \mathrm{C} 7,1}$. In order to test the validity of these assumptions in the other flames, the ratio of the root-mean-square of the residual (for the fuel equation) to the root-mean square of the fuel burning rate ("Src" term in Fig. 2) at the location (in cspace) of peak fuel burning rate $\left(c_{\text {peak }}\right)$ is plotted in Fig. 3 as a function of $K a_{\delta}$. A small ratio suggests that the assumptions are valid and, although the flames are highly three-dimensional and unsteady (in physical space), they can be well described by the one-dimensional (in phase space) flamelet equations (Eq. 12). This is the case for flames $\mathrm{A}_{n \mathrm{C} 7,1}, \mathrm{~B}_{n \mathrm{C} 7,1}, \mathrm{~B}_{\mathrm{CH} 4,1}$, and $\mathrm{B}_{\mathrm{ACH} 3,1}$. For cases $\mathrm{C}_{n \mathrm{C} 7,1}$ and $\mathrm{D}_{n \mathrm{C} 7,1}$, the ratio is relatively large suggesting the departure from a local flamelet structure. This is not surprising as in these flames the reaction zones are thickened or distributed, as shown in Lapointe et al. [8]. These results suggest that, given the current definition used for the Karlovitz number at the reaction zone, the flamelet equations are valid up to $K a_{\delta} \approx 10$.

[Fig. 3 about here.] 


\subsection{Dependence of the fuel burning rate on $c$ and $\chi$}

Given the validity of the flamelet equations (as shown in the previous section), the fuel burning rate should solely be a function of the progress variable $c$ and its dissipation rate $\chi$. Figure 4 presents the joint probability density function of the fuel consumption rate and the dissipation rate of the progress variable in flame $\mathrm{B}_{n \mathrm{C} 7,1}$, both of which are evaluated at $c_{\text {peak }}\left(c_{\text {peak }}=0.157\right)$. The correlation coefficients are listed in Table 4 and are compared to those obtained with strain rate and curvature (taken from Ref. [7]). This figure should be contrasted with Fig. 12 and 13 shown in Ref [7]. As a reference, the normalized (by flame quantities) strain rate, dissipation rate, and curvature take the following values: $a_{t} l_{F} / S_{L}$ ranges from -30 to 90 approximately, $\chi l_{F} / S_{L}$ ranges from 0 to 1.5 approximately, and $\kappa l_{F}$ ranges from approximately -6 to 6 .

It is clear from Fig. 4 and Table 4 that the fuel burning rate at $c_{\text {peak }}$ is far more correlated with $\chi$ than curvature or tangential strain rate $a_{t}$. Similar observations were made by Lapointe and Blanquart [9] who also found the fuel burning rate to be strongly correlated with dissipation rate at $T_{\text {peak }}$ for case $\mathrm{B}_{n \mathrm{C} 7,1}, \mathrm{~B}_{\mathrm{CH} 4,1}$, and $\mathrm{B}_{\mathrm{ACH} 3,1}$ and to a lesser extent for cases $\mathrm{C}_{n \mathrm{C} 7,1}$ and $\mathrm{D}_{n \mathrm{C} 7,1}$. Physically, this means that turbulence affects the fuel burning rate by compressing or expanding the isosurfaces of the progress variable. It also means that straining the flow field is not equivalent to straining these isosurfaces in the context of high Karlovitz premixed flames. Note that the correlation with $\chi$ is comparatively good for other values of $c$. For instance, at $c=0.13$, which corresponds to the location of approximately half the peak burning rate in a laminar flame, the correlation coefficients are above 0.98 (case $\mathrm{B}_{n \mathrm{C} 7,1}$ ). 
[Fig. 4 about here.]

[Table 4 about here.]

This result suggests that there should exist a function $f(c, \chi)$ that estimates accurately the local fuel burning rate. Such a function can be easily identified a posteriori, i.e. using the data from the DNS. We first define the prediction error made by the function $f$ by the following $L_{2}$-norm over the whole domain:

$$
\epsilon_{f}=\left(\frac{\int_{t_{1}}^{t_{2}} \int_{\Omega}\left(f-\dot{\omega}_{F}\right)^{2} \mathrm{~d} V \mathrm{~d} t}{\int_{t_{1}}^{t_{2}} \int_{\Omega} \dot{\omega}_{F}^{2} \mathrm{~d} V \mathrm{~d} t}\right)^{1 / 2} .
$$

A similar approach was used to quantify the error made by LES filters on chemical source terms [47] and by scalar dissipation rate sub-grid-scale models [48]. The function that minimizes this error, given that $f$ is a function of $c$ and $\chi$ only, also called the optimal estimator $[49,48]$, is the mean of the fuel burning rate conditional on $c$ and $\chi$, i.e.

$$
\underset{f(c, \chi)}{\arg \min } \epsilon_{f}=<\dot{\omega}_{F} \mid c, \chi>_{V},
$$

where the subscript $V$ references to a volumetric, as opposed to surfacic average. More details on optimal estimation can be found in Ref. [49].

The optimal estimator given by Eq. 14 is evaluated for each of the six unity Lewis number turbulent flames. Details on how the conditional means are computed discretely can be found in the supplemental material. Note that 300 bins per direction are used for the one- and two-dimensional conditional means, whereas 100 bins are used in the next section for the three-dimensional conditional means (it is shown in the supplemental material that this number is sufficient to obtain converged statistics). The prediction errors are listed in Tables 5 and 6 . They are compared to the prediction errors made by the 
following functions: $\left\langle\dot{\omega}_{F} \mid c\right\rangle_{V}$ (optimal estimator given that $f$ is a function of the progress variable only), $\left\langle\dot{\omega}_{F}\right| c, \kappa>_{V}$ (optimal estimator given that $f$ is a function of the progress variable and the curvature only), and $<\dot{\omega}_{F} \mid c, a_{t}>_{V}$ (optimal estimator given that $f$ is a function of the progress variable and the tangential strain rate only).

First, it is clear from Tables 5 and 6 that, allowing a dependence on $c$ and $\chi$ instead of $c$ only improves dramatically the prediction of the local, instantaneous fuel burning rate for cases A and B. This is illustrated in Fig. 5 which shows the probability density function of the point-wise comparison between the predicted and the actual fuel burning rate (case $\left.\mathrm{B}_{n \mathrm{C} 7,1}\right)$. For cases $\mathrm{C}$ and $\mathrm{D}$, the improvement is much less significant (in relative value). Considering the results from the previous section, as $K a_{\delta}$ increases, the local structure of the reaction zone departs from that of flamelets and hence, locally, the burning rate fluctuations cannot be solely attributed to fluctuations in the dissipation rate.

Second, the choice of the second variable has a strong effect on the prediction error (Tables 5 and 6). Consistently with the results of Table 4, the fuel burning rate is much better approximated by the progress variable dissipation rate than by either curvature or tangential strain rate.

[Table 5 about here.]

[Table 6 about here.]

[Fig. 5 about here.]

[Fig. 6 about here.] 
Finally, the prediction errors obtained with both $\left\langle\dot{\omega}_{F}\right| c>_{V}$ and $\left\langle\dot{\omega}_{F} \mid c, \chi\right\rangle_{V}$ are plotted as a function of the Karlovitz number at the reaction zone in Fig. 6 . This figure clearly illustrates the quantitative improvement in the prediction of the fuel burning rate by considering the dissipation rate as a second variable and how this improvement depends on the Karlovitz number.

Note that the prediction error with $\left\langle\dot{\omega}_{F} \mid c\right\rangle_{V}$ is significantly smaller for the methane/air flame than for all the large hydrocarbon/air flames. The cause of this difference should be investigated in future work.

In summary, the optimal estimator $\left\langle\dot{\omega}_{F} \mid c, \chi\right\rangle_{V}$ provides a very good estimation of the instantaneous, local fuel burning rate up to $K a_{\delta} \approx 10$. The results from this section show that $\dot{\omega}_{F}(\mathbf{x}, t)$, which lives in a $N$-dimensional phase-space (with $N=17-74$ in the present flames), collapses very nicely on a two-dimensional manifold for $K a_{\delta}<10$.

\section{$5 \quad$ Non-unity Lewis numbers}

The previous section provided a framework that was used to physically and mathematically explain the effect of turbulence on the fuel burning rate, in the absence of differential diffusion. In the present section, effects of differential diffusion are discussed.

\subsection{Validity of the flamelet equations}

Similar to Section 4.1, to test the validity of the two assumptions made in this section, the budget of Eq. 9 from the DNS is shown and compared to the 
corresponding unstretched laminar solution in Fig. 7 for flame $\mathrm{B}_{n \mathrm{C} 7}$.

[Fig. 7 about here.]

[Fig. 8 about here.]

[Fig. 9 about here.]

First and in contrast with the unity Lewis number limit, non-zero terms are found outside the reaction zone for $n-\mathrm{C}_{7} \mathrm{H}_{16}$ and $\mathrm{H}_{2} \mathrm{O}$, namely the diffusion term and the differential diffusion-induced convective term. This is observed in both the laminar and turbulent flames. It is also worth noting that the terms associated with the velocity correction are still negligible for all species considered.

Second and also in contrast with the unity Lewis number limit, the conditional mean of each term departs from the laminar solution. Three important observations are listed below.

- The absolute values for each term are significantly reduced from those of the laminar flame.

- However, when normalized with the respective maximum values both the laminar and turbulent flames show similar mean flamelet structures, as shown in Fig 8. In other words, the relative magnitudes of the terms in the flamelet equations are essentially unchanged from the laminar case.

- Nevertheless, the peak of each term is shifted towards higher $c$-values, consistent with the disappearance of differential diffusion effects (going towards $L e=1)$ in the presence of turbulence $[11,50,6,8]$. 
Third, and most importantly, the residuals (Eq. A.13 in Appendix A) remain relatively small (both the mean and standard deviation) compared to the other terms, although they are not completely negligible throughout the flame. Recalling that the preheat zone is largely thickened in the turbulent flames, it is not expected that the assumptions made in this section are valid throughout this preheat zone. More quantitatively and similar to Fig. 3 for the unity Lewis number limit, Fig. 9 shows that the residuals are relatively small at the reaction zones for cases AAA to B and significantly larger for cases C and D. As in the unity Lewis number limit, these results suggest that the flamelet equations are valid for $K a_{\delta}<10$. For these flames, the residuals remain sufficiently small in the reaction zone to expect a strong dependence of the source terms on $c, \chi$, and $\xi$, which will be evaluated in the next section.

\subsection{Dependence of the burning rate on $c, \chi$, and $\xi$}

As in Section 4.3, the joint probability density function of the fuel burning rate (at $c_{\text {peak }}$ ) and dissipation rate is presented in Fig. 10 for flame $\mathrm{B}_{n \mathrm{C} 7}$. Note that $c_{\text {peak }}$ corresponds to the location in $c$-space of the peak mean (conditional on $c$ ) burning rate in the DNS $\left(c_{\text {peak }}=0.127\right)$. In contrast, $c_{\text {lam,peak }}$ is the equivalent for a one-dimensional unstretched flame $\left(c_{\text {lam,peak }}=0.117\right)$. Note that this distinction is important only in the non-unity Lewis number case.

Table 7 compares the correlation coefficients for Fig. 10 to those obtained with strain rate and curvature. The fuel burning rate is, as in the unity Lewis number case, more correlated with the dissipation rate than the two other variables. However, this correlation was significantly stronger in the unity Lewis number case than in the present non-unity Lewis number case (which 
takes the form of a wider spread in Fig. 10). Given the results of the last section, it is expected that the spread seen in Fig. 10 will be reduced by allowing a dependence on $\xi$.

[Table 7 about here.]

[Fig. 10 about here.]

In other words, the optimal estimator given, $c, \chi$, and $\xi$ should predict the fuel burning rate significantly better than the optimal estimator given, $c$ only or $c$ and $\chi$.

Similar to Fig. 5, Fig. 11 shows the probability density function of the pointwise comparison in flame $\mathrm{B}_{n \mathrm{C} 7}$ between the predicted and the actual fuel burning rate, for three optimal estimators: $\left\langle\dot{\omega}_{F}\right| c>_{V},\left\langle\dot{\omega}_{F}\right| c, \chi>_{V}$, and $<\dot{\omega}_{F} \mid c, \chi, \xi>_{V}$

[Fig. 11 about here.]

The prediction errors for these functions, in addition to $\left\langle\dot{\omega}_{F}\right| c, \chi, \kappa>_{V}$ and $<\dot{\omega}_{F} \mid c, \chi, \xi+\rho \kappa\left(\frac{\chi \alpha}{2}\right)^{1 / 2}>_{V}$, where $\xi+\rho \kappa\left(\frac{\chi \alpha}{2}\right)^{1 / 2}$ is the diffusion rate $\xi$ minus the curvature term as found in the flamelet equations of Lodier et al. [38], are listed in Tables 8 and 9. Table 8 includes all the non-unity Lewis number large hydrocarbon/air flames, whereas the methane/air non-unity Lewis number flames are included in Table 9. Three major observations are discussed below.

First, for the lower- $K a$ flames (Table 8 ), $<\dot{\omega}_{F} \mid c, \chi, \xi>_{V}$ provides a very good estimate of the fuel burning rate. In particular, the prediction error is more than 10 times smaller than with $<\dot{\omega}_{F} \mid c>_{V}$ for case $\mathrm{AAA}_{n \mathrm{C}}$. As expected, as the Karlovitz number at the reaction zone is increased, these 
errors increase. This is illustrated in Fig. 12 which presents the estimation error for $<\dot{\omega}_{F} \mid c, \chi, \xi>_{V}$ (and $<\dot{\omega}_{F} \mid c>_{V}$ ) as a function of $K a_{\delta}$. Once again, and as mentioned in Section 5.1, the departure from a local flamelet structure is non-negligible in the higher- $K a$ flames. In addition, at constant $K a_{\delta}$, the prediction errors for $\left\langle\dot{\omega}_{F}\right| c, \chi, \xi>_{V}$ are essentially unchanged between the different flames considered (in which different fuels, unburnt temperatures, pressures, configurations and integral length scale to laminar flame thickness ratios were considered), with the exception of slightly lower values for the methane/air flames.

[Table 8 about here.]

[Table 9 about here.]

[Fig. 12 about here.]

Second, the prediction error obtained with $<\dot{\omega}_{F} \mid c, \chi, \xi>_{V}$ is significantly smaller than with $\left\langle\dot{\omega}_{F}\right| c, \chi, \kappa>_{V}$, in all the large hydrocarbon/air flames considered (Table 8) and it is consistently smaller (to a lesser extent) in the methane/air flames (Table 9). As predicted by the flamelet equations and as discussed in this section, $\xi$ is a more relevant parameter than $\kappa$.

Third, the prediction error obtained with $<\dot{\omega}_{F} \mid c, \chi, \xi>_{V}$ is consistently smaller than with $<\dot{\omega}_{F} \mid c, \chi, \xi+\rho \kappa\left(\frac{\chi \alpha}{2}\right)^{1 / 2}>_{V}$ in all the flames considered. The relative difference is particularly important in the lower- $K a$ flames. This result shows that the curvature term should not be neglected in Eq. 11 .

Note that, similar to the unity Lewis number case, the prediction error with $<\dot{\omega}_{F} \mid c>_{V}$ is significantly smaller for the non-unity Lewis number methane/air flames than for all the large hydrocarbon/air flames. The cause of this differ- 
ence should be investigated in future work.

To summarize, the importance of $\chi$ and $\xi$ as opposed to $a_{t}$ and $\kappa$ was demonstrated for a wide range of turbulent premixed flames. In particular, it was shown that the optimal estimator $\left\langle\dot{\omega}_{F}\right| c, \chi, \xi>_{V}$ can predict the instantaneous, local fuel burning rate particularly well for moderate $K a_{\delta}$ (less than 10).

\section{Summary and conclusions}

The validity of premixed flamelet equations and the dependence of the fuel burning rate on the parameters involved in these equations were investigated on a large series of direct numerical simulations of turbulent premixed flames in the TRZ and the DRZ regimes. Methane, toluene, $n$-heptane, and iso-octane were the fuels considered over the following range of unburnt conditions and turbulence characteristics: $T_{u}=298-800 \mathrm{~K}, P_{0}=0.1-2 \mathrm{MPa}, \phi=0.7-0.9$, $u^{\prime} / S_{L}=2.3-60$, and $l / l_{F}=0.9-4.0$. Two flow configurations were used $[6,39]$ and all the simulations were performed with detailed chemistry (17-74 species and $17-976$ reactions). Flames with unity and non-unity Lewis numbers were considered separately to isolate turbulence-chemistry interaction from differential diffusion effects.

In the unity Lewis number limit, the flamelet equations involve a single parameter, the dissipation rate of the progress variable $\chi$. First, the flamelet equations were found to be valid for all flames in the TRZ regime, more precisely for Karlovitz numbers at the reaction zone smaller than 10. Second, a consequence from this result is that the fuel burning rate correlates with $\chi$ 
significantly better than with curvature or strain rate. In particular, the fuel burning rate $\dot{\omega}_{F}$ can be approximated accurately by $\left\langle\dot{\omega}_{F}\right| c, \chi>_{V}$ in the TRZ regime.

In the non-unity Lewis number case, the flamelet equations involve an additional parameter, the diffusion rate of the progress variable $\xi$. First, while differential diffusion causes a larger departure from the flamelet equations, the flames in the TRZ regime were found to be still well described by the these equations (with $K a_{\delta}<10$ ). Second, the importance of considering $\xi$ instead of curvature was highlighted. Finally, the fuel burning rate $\dot{\omega}_{F}$ can be approximated accurately by $\left\langle\dot{\omega}_{F}\right| c, \chi, \xi>_{V}$ in the TRZ regime. This optimal estimator predicts the fuel burning rate up to an order of magnitude better than the mean burning rate $<\dot{\omega}_{F} \mid c>_{V}$.

Finally, the cause for the lower prediction error with $\left\langle\dot{\omega}_{F}\right| c>_{V}$ in the methane/air flames (vs. the large hydrocarbon/air flames) cannot be solely attributed to differential diffusion and should be investigated in future work. This difference emphasizes the importance of considering large hydrocarbon fuels, as opposed to methane, to study premixed turbulent flames relevant to combustion in transportation engines.

\section{Acknowledgments}

The authors gratefully acknowledge funding from the Air Force Office of Scientific Research (Award FA9550-12-1-0144) under the supervision of Dr. Chiping Li, the Fonds de recherche du Québec - Nature et technologies (Postdoctoral fellowship D3), the Academic Computer Center in Gdańsk (CI TASK), the 
Social Found of the European Union (Scholarship for Visiting Professor at the Warsaw University of Technology), the U.S. Department of Energy-Basic Energy Sciences (DE-SC006591) under the supervision of Dr. Wade Sisk, and the Natural Sciences and Engineering Research Council of Canada (NSERC Postgraduate Scholarship D). The authors would also like to thank Dr. Simon Lapointe for his help in processing the data and Dr. Ramanan Sankaran, Dr. Evatt R. Hawkes, Dr. Chun Sang Yoo, Dr. Jacqueline H. Chen, along with Dr. Hemanth Kolla who shared with the authors the DNS data of Ref [39].

\section{A Residuals from the flamelet equations}

The residuals, labeled as "Res", are defined as the missing terms in the flamelet equations compared to the full transformed species transport equations, i.e. such that

$$
\text { Conv }+ \text { Diff }+ \text { Src }+ \text { DiffConv }+ \text { Velcor }+ \text { Res }=0 .
$$

In the non-unity Lewis number case,

$$
\begin{gathered}
\text { Conv }=-\dot{\omega}_{c} \frac{\mathrm{d} Y_{i}}{\mathrm{~d} c}, \\
\operatorname{Diff}=\frac{\rho \chi}{2 L e_{i}} \frac{\mathrm{d}^{2} Y_{i}}{\mathrm{~d} c^{2}}, \\
\operatorname{Src}=\dot{\omega}_{i},
\end{gathered}
$$

DiffConv $=$

$$
\begin{gathered}
{\left[\xi\left(\frac{1}{L e_{i}}-\frac{1}{L e_{c}}\right)+\frac{\rho \chi}{2} \frac{\mathrm{d}}{\mathrm{d} c}\left(\frac{1}{L e_{i}}-\frac{1}{L e_{c}}\right)\right] \frac{\mathrm{d} Y_{i}}{\mathrm{~d} c},} \\
\text { Velcor }=\nabla \cdot\left(\rho Y_{i} \mathbf{V}_{\mathbf{c}, \mathbf{i}}\right)-\sum_{j=1}^{N} b_{j} \nabla \cdot\left(\rho Y_{j} \mathbf{V}_{\mathbf{c}, \mathbf{j}}\right) \frac{\mathrm{d} Y_{i}}{\mathrm{~d} c},
\end{gathered}
$$


and

$$
\begin{gathered}
\text { Res }=-\rho \frac{\partial Y_{i}}{\partial \tau}-\underbrace{\rho \sum_{k=2}^{3}\left[\frac{\partial Y_{i}}{\partial c_{k}}\left(\frac{\partial c_{k}}{\partial t}+\mathbf{u} \cdot \nabla c_{k}\right)\right]}_{\text {Lagrangian transport }} \\
+\underbrace{\sum_{k=2}^{3} \frac{\rho \chi_{k}}{2 L e_{i}} \frac{\partial^{2} Y_{i}}{\partial c_{k}^{2}}+\frac{2 \rho \alpha}{L e_{i}}\left(\nabla c_{2} \cdot \nabla c_{3}\right) \frac{\partial^{2} Y_{i}}{\partial c_{2} \partial c_{3}}}_{\text {tangential diffusion }} \\
+\underbrace{\nabla \cdot\left(\frac{\rho \alpha}{L e_{i}} \nabla c_{2}\right) \frac{\partial Y_{i}}{\partial c_{2}}+\nabla \cdot\left(\frac{\rho \alpha}{L e_{i}} \nabla c_{3}\right) \frac{\partial Y_{i}}{\partial c_{3}}}_{\text {tangential convection }} .
\end{gathered}
$$

In the unity Lewis number case,

$$
\begin{gathered}
\text { Conv }=-\dot{\omega}_{c} \frac{\mathrm{d} Y_{i}}{\mathrm{~d} c}, \\
\text { Diff }=\frac{\rho \chi}{2} \frac{\mathrm{d}^{2} Y_{i}}{\mathrm{~d} c^{2}} \\
\text { Src }=\dot{\omega}_{i}, \\
\text { DiffConv }=0, \\
\text { Velcor }=0,
\end{gathered}
$$

and

$$
\begin{gathered}
\operatorname{Res}=-\rho \frac{\partial Y_{i}}{\partial \tau}-\underbrace{\rho \sum_{k=2}^{3}\left[\frac{\partial Y_{i}}{\partial c_{k}}\left(\frac{\partial c_{k}}{\partial t}+\mathbf{u} \cdot \nabla c_{k}\right)\right]}_{\text {Lagrangian transport }} \\
+\underbrace{\sum_{k=2}^{3} \frac{\rho \chi_{k}}{2} \frac{\partial^{2} Y_{i}}{\partial c_{k}^{2}}+2 \rho \alpha\left(\nabla c_{2} \cdot \nabla c_{3}\right) \frac{\partial^{2} Y_{i}}{\partial c_{2} \partial c_{3}}}_{\text {tangential diffusion }} \\
+\underbrace{\nabla \cdot\left(\rho \alpha \nabla c_{2}\right) \frac{\partial Y_{i}}{\partial c_{2}}+\nabla \cdot\left(\rho \alpha \nabla c_{3}\right) \frac{\partial Y_{i}}{\partial c_{3}}}_{\text {tangential convection }} .
\end{gathered}
$$

More details on the transformed species transport equations can be found in [37], Section 7.3. 


\section{References}

[1] D. Linse, C. Hasse, B. Durst, An experimental and numerical investigation of turbulent flame propagation structure in a turbo-charged direct injection gasoline engine, Combust. Theory Model. 13 (1) (2009) 167-188.

[2] C. Mounam-Rousselle, L. Landry, F. Halter, F. Foucher, Experimental characteristics of turbulent premixed flame in a boosted Spark-Ignition engine, Proc. Comb. Inst. 34 (1) (2013) 2941-2949.

[3] J. Zhou, The study of the oxygen controlled combustion in downsized SI engine, Ph.D. thesis, Université dOrléans (2013).

[4] Y. Huang, Modeling and simulation of combustion dynamics in lean-premixed swirl-stabilized gas-turbine engines, Ph.D. thesis, Pennsylvania State University (2003).

[5] W.-W. Kim, S. Menon, Numerical modeling of turbulent premixed flames in the thin-reaction-zones regime, Comb. Sci. Tech. 160 (1) (2000) 119-150.

[6] B. Savard, B. Bobbitt, G. Blanquart, Structure of a high Karlovitz ${ }_{n}$ - $\mathrm{C}_{7} \mathrm{H}_{16}$ premixed turbulent flame, Proc. Comb. Inst. 35 (2015) 1377-1384.

[7] B. Savard, G. Blanquart, Broken reaction zone and differential diffusion effects in high Karlovitz $n$ - $\mathrm{C}_{7} \mathrm{H}_{16}$ premixed turbulent flames, Combust. Flame 162 (5) (2015) 2020-2033.

[8] S. Lapointe, B. Savard, G. Blanquart, Differential diffusion effects, distributed burning, and local extinctions in high karlovitz premixed flames, Combust. Flame 162 (2015) 3341-3355.

[9] S. Lapointe, G. Blanquart, Fuel and chemistry effects in high karlovitz premixed turbulent flames, Combust. Flame 167 (2016) 294-307. 
[10] B. Savard, S. Lapointe, A. Teodorczyk, Numerical investigation of the effect of pressure on heat release rate in iso-octane premixed turbulent flames under conditions relevant to SI engines, Proc. Comb. Inst. (2016) http://dx.doi.org/10.1016/j.proci.2016.07.056.

[11] N. Peters, Turbulent Combustion, Cambridge University Press, Cambridge, 2000.

[12] T. Wabel, A. Skiba, J. Temme, J. Driscoll, Measurements to determine the regimes of turbulent premixed flames, in: 51st AIAA/ ASME/ SAE/ ASEE Joint Propulsion Conference, Orlando, USA, 2015.

[13] D. Haworth, T. Poinsot, Numerical simulations of Lewis number effects in turbulent premixed flames, J. Fluid Mech. 244 (1992) 405-436.

[14] J. Chen, H. Im, Correlation of flame speed with stretch in turbulent premixed methane/air flames, Twenty-Seventh Symposium (International) on Combustion 1-2 (1998) 819-826.

[15] J. Chen, H. Im, Stretch effects on the burning velocity of turbulent premixed hydrogen/air flames, Proc. Comb. Inst. 28 (2000) 211-218.

[16] E. Hawkes, J. Chen, Comparison of direct numerical simulation of lean premixed methane-air flames with strained laminar flame calculations, Combust. Flame 144 (2006) 112-125.

[17] D. Bradley, P. Gaskell, X. Gu, A. Sedaghat, Premixed flamelet modelling: Factors influencing the turbulent heat release rate source term and the turbulent burning velocity, Combust. Flame 143 (2005) 227-245.

[18] D. Bradley, M. Lawes, K. Liu, M. Mansour, Measurements and correlations of turbulent burning velocities over wide ranges of fuels and elevated pressures, Proc. Comb. Inst. 34 (2013) 1516-1526. 
[19] E. Knudsen, H. Kolla, E. Hawkes, H. Pitsch, LES of a premixed jet flame DNS using a strained flamelet model, Combust. Flame 160 (2013) 29112927.

[20] K. Bray, The interaction between turbulence and combustion, Seventeenth Symposium (International) on Combustion (1979) 223-233.

[21] A. N. Lipatnikov, S. Nishiki, T. Hasegawa, DNS assessment of relation between mean reaction and scalar dissipation rates in the flamelet regime of premixed turbulent combustion, Combust. Theory Model. 19 (3) (2015) 309-328.

[22] N. Swaminathan, K. Bray, Scalar dissipation and mean reaction rates in premixed turbulent combustion, Combust. Flame 143 (2005) 549-565.

[23] K. Bray, M. Champion, P. Libby, N. Swaminathan, Scalar dissipation and mean reaction rates in premixed turbulent combustion, Combust. Flame 158 (2011) 2017-2022.

[24] Y. Gao, N. Chakraborty, N. Swaminathan, Scalar dissipation rate transport and in the context of large eddy simulations for turbulent premixed flames with non-unity Lewis number, Flow Turbul. Combust. 93 (2014) 461-486.

[25] Y. Gao, N. Chakraborty, N. Swaminathan, Algebraic closure of scalar dissipation rate for large eddy simulations of turbulent premixed combustion, Combust. Sci. Technol. 186 (2014) 1309-1337.

[26] Y. Gao, N. Chakraborty, N. Swaminathan, Scalar dissipation rate transport and its modeling for large eddy simulations of turbulent premixed flames, Combust. Sci. Technol. 187 (2015) 362-383.

[27] R. Bilger, Conditional moment closure for turbulent reacting flow, Phys. Fluids A 5 (1993) 436-444.

[28] S. Amzin, N. Swaminathan, J. Rogerson, J. Kent, Conditional moment closure for turbulent premixed flames, Combust. Sci. Technol 184 (2012) 1743-1767. 
[29] D. Veynate, L. Vervisch, Turbulent combustion modeling, Prog. Energy Combust. Sci. 28 (2002) 193-266.

[30] H. Kolla, Scalar dissipation rate based flamelet modelling of turbulent premixed flames, Ph.D. thesis, University of Cambridge (2009).

[31] P. Nguyen, L. Vervisch, V. Subramanian, P. Domingo, Multidimensional flamelet-generated manifolds for partially premixed combustion, Combust. Flame 157 (2010) 43-61.

[32] C. Kortschik, S. Honnet, N. Peters, Influence of curvature on the onset of autoignition in a corrugated counterflow mixing field, Combust. Flame 142 (12) (2005) 140-152.

[33] H. Xu, F. Hunger, M. Vascellari, C. Hasse, A consistent flamelet formulation for a reacting char particle considering curvature effects, Combust. Flame 160 (11) (2013) 2540-2558.

[34] Y. Xuan, G. Blanquart, M. E. Mueller, Modeling curvature effects in diffusion flames using a laminar flamelet model, Combust. Flame 161 (5) (2014) 12941309.

[35] A. Scholtissek, W. L. Chan, H. Xu, F. Hunger, H. Kolla, J. H. Chen, M. Ihme, C. Hasse, A multi-scale asymptotic scaling and regime analysis of flamelet equations including tangential diffusion effects for laminar and turbulent flames, Combust. Flame 162 (4) (2015) 1507-1529.

[36] H. Pitsch, N. Peters, A consistent flamelet formulation for non-premixed combustion considering differential diffusion effects, Combust. Flame 114 (1-2) (1998) 26-40.

[37] B. Savard, Characterization and modeling of premixed turbulent $n$-heptane flames in the thin reaction zone regime, Ph.D. thesis, California Institute of Technology (2015). 
[38] G. Lodier, L. Vervisch, V. Moureau, P. Domingo, Composition-space premixed flamelet solution with differential diffusion for in situ flamelet-generated manifolds, Combust. Flame 158 (10) (2011) 2009-2016.

[39] R. Sankaran, E. Hawkes, C. Yoo, J. Chen, Response of flame thickness and propagation speed under intense turbulence in spatially developing lean premixed methane-air jet flames, Combust. Flame 162 (2015) 3294-3306.

[40] P. Curran, H.J. Gaffuri, W. Pitz, C. Westbrook, A comprehensive modeling study of $n$-heptane oxidation, Combust. Flame 114 (1998) 149-177.

[41] M. Mehl, W. J. Pitz, C. K. Westbrook, H. J. Curran, Kinetic modeling of gasoline surrogate components and mixtures under engine conditions, Proc. Comb. Inst. 33 (2011) $193-200$.

[42] K. Niemeyer, C. Sung, Mechanism reduction for multicomponent surrogates: A case study using toluene reference fuels, Combust. Flame 161 (11) (2014) $2752-2764$.

[43] P. Dagaut, M. Cathonnet, The ignition, oxidation, and combustion of kerosene: A review of experimental and kinetic modeling, Prog. Energy Combust. Sci. 32 (2006) 48-92.

[44] B. Bobbitt, S. Lapointe, G. Blanquart, Vorticity transformation in high karlovitz number premixed flames, Phys. Fluids 28 (2016) 015101.

[45] S. Lapointe, Simulation of premixed hydrocarbon flames at high turbulence intensities, Ph.D. thesis, California Institute of Technology (2016).

[46] R. W. Bilger, The structure of diffusion flames, Comb. Sci. Tech. 13 (1976) $155-170$.

[47] S. Mukhopadhyay, Modeling turbulent combustion using filtered flamelets, Dissertation, Technische Universiteit Eindhoven (2014). 
[48] G. Balarac, H. Pitsch, V. Raman, Modeling of the subfilter scalar dissipation rate using the concept of optimal estimators, Phys. Fluids 20 (2008) 091701.

[49] A. Moreau, O. Teytaud, J. Bertoglio, Optimal estimation for large-eddy simulation of turbulence and application to the analysis of subgrid models, Phys. Fluids 18 (2006) 105101.

[50] B. Savard, G. Blanquart, An a priori model for the effective species lewis numbers in premixed turbulent flames, Combust. Flame 161 (2014) 1547-1557.

[51] H. Pitsch, Flamemaster: A C++ computer program for 0D combustion and 1D laminar flame calculations. Available at http://www.itv.rwthaachen.de/downloads/flamemaster/. (1998).

[52] K. Pearson, Notes on regression and inheritance in the case of two parents, P. R. Soc. London 58 (1895) 240-242.

[53] G. Székely, M. Rizzo, N. Bakiroz, Measuring and testing dependence by correlation of distances, Ann. Stat. 35 (2007) 2769-2794. 


\section{List of Figures}

$1 \quad$ Location of the DNS considered in Peter's regime diagram [11].

The color code for each fuel is maintained throughout this paper.

2 Mean of the terms in Eq. 12 conditioned on $c$ for flame $\mathrm{B}_{n \mathrm{C} 7,1},<\operatorname{term} \mid c>$ (solid lines). The bars correspond to the standard deviation of these terms conditioned on $c$, $<(\text { term }-<\operatorname{term} \mid c>)^{2} \mid c>^{1 / 2}$. The red line corresponds to the residual of the respective equations (Eq. A.13 in Appendix A). The labels Conv, Diff, Src, and Res are used for the convection, diffusion, chemical source, and residual terms respectively. For clarity, a description of all the terms is provided in Appendix A. The dashed lines are taken from the corresponding one-dimensional unstretched laminar flame. Each term is normalized by the absolute value of the largest term (chemical source term) in the laminar flame. Note that the conditional means are evaluated as in Lapointe et al. [8].

3 Ratio of the conditional RMS of the residuals from the flamelet equation (fuel equation) to the conditional RMS of the fuel burning rate at $c=c_{\text {peak }}$ as a function of the Karlovitz number at the reaction zone for the unity Lewis number flames.

Dashed lines are included to guide the reader with respect to the discussion in the main text. 
4 Joint PDF of the fuel burning rate and the dissipation rate of the progress variable, normalized by their laminar values, on the isosurface of $c=c_{\text {peak }}$ in flame $\mathrm{B}_{n \mathrm{C} 7,1}$.

5 Joint PDF of the predicted fuel burning rate and the actual burning rate in flame $\mathrm{B}_{n \mathrm{C} 7,1}$.

6 Prediction error for $<\dot{\omega}_{F} \mid c>_{V}$ (filled symbols) and $<\dot{\omega}_{F} \mid c, \chi>_{V}$ (open symbols) as a function of the Karlovitz number at the reaction zone for all the unity Lewis number flames.

7 Mean of the terms in Eq 9 conditioned on $c$ for flame $\mathrm{B}_{n \mathrm{C} 7}$, $<\operatorname{term} \mid c>$. The bars correspond to the standard deviation of these terms conditioned on $c,<(\text { term }-<\operatorname{term} \mid c>)^{2} \mid c>^{1 / 2}$. The red dashed line corresponds to the residual of the respective equations (Eq. A.7 in Appendix A). The labels Conv, Diff, Src, Diff-Conv, Vel cor, and Res are used for the convection, diffusion, chemical source, differential diffusion-induced convection, velocity correction, and residual terms respectively. For clarity, a description of all the terms is provided in Appendix A. Each term is normalized by the absolute value of the largest term (chemical source term) in the corresponding one-dimensional unstretched laminar flame. Note that the conditional means are evaluated as in Lapointe et al. [8]. 
8 Mean profiles from Fig. 7 for the $n-\mathrm{C}_{7} \mathrm{H}_{16}$ flamelet equation normalized to unity in both the laminar and turbulent cases. The dashed and solid lines correspond to the laminar and turbulent flames, respectively.

9 Ratio of the conditional RMS of the residuals from the flamelet equation (fuel equation) to the conditional RMS of the fuel burning rate at $c=c_{\text {peak }}$ as a function of the Karlovitz number at the reaction zone for the non-unity Lewis number flames. Dashed lines are included to guide the reader with respect to the discussion in the main text.

10 Joint PDF of fuel burning rate and dissipation rate of the progress variable, normalized by their laminar values (at $\left.c=c_{\text {lam,peak }}\right)$, on the isosurface of $c=c_{\text {peak }}$ in flame $\mathrm{B}_{n \mathrm{C} 7}$.

11 Joint PDF of the predicted fuel burning rate and the actual burning rate in flame $\mathrm{B}_{n \mathrm{C} 7}$.

12 Prediction error for $<\dot{\omega}_{F} \mid c>_{V}$ (filled symbols) and $<\dot{\omega}_{F} \mid c, \chi, \xi>_{V}$ (open symbols) as a function of the Karlovitz number at the reaction zone for all the non-unity Lewis number flames. 


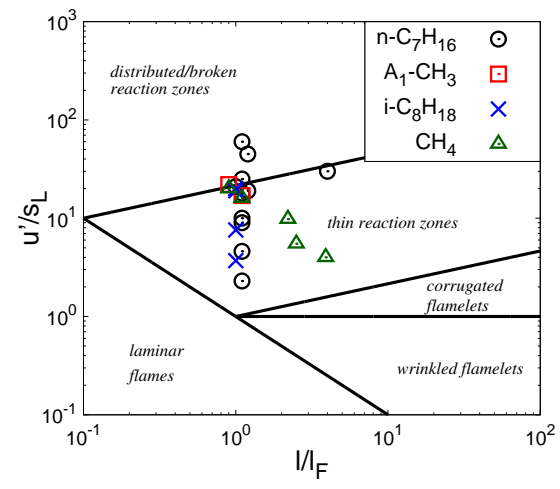

Fig. 1. Location of the DNS considered in Peter's regime diagram [11]. The color code for each fuel is maintained throughout this paper. 
Fig. 2. Mean of the terms in Eq. 12 conditioned on $c$ for flame $\mathrm{B}_{n \mathrm{C} 7,1},<$ term $|c\rangle$ (solid lines). The bars correspond to the standard deviation of these terms conditioned on $c,<(\text { term }-<\operatorname{term} \mid c>)^{2} \mid c>^{1 / 2}$. The red line corresponds to the residual of the respective equations (Eq. A.13 in Appendix A). The labels Conv, Diff, Src, and Res are used for the convection, diffusion, chemical source, and residual terms respectively. For clarity, a description of all the terms is provided in Appendix A. The dashed lines are taken from the corresponding one-dimensional unstretched laminar flame. Each term is normalized by the absolute value of the largest term (chemical source term) in the laminar flame. Note that the conditional means are evaluated as in Lapointe et al. [8]. 
Fig. 3. Ratio of the conditional RMS of the residuals from the flamelet equation (fuel equation) to the conditional RMS of the fuel burning rate at $c=c_{\text {peak }}$ as a function of the Karlovitz number at the reaction zone for the unity Lewis number flames. Dashed lines are included to guide the reader with respect to the discussion in the main text. 


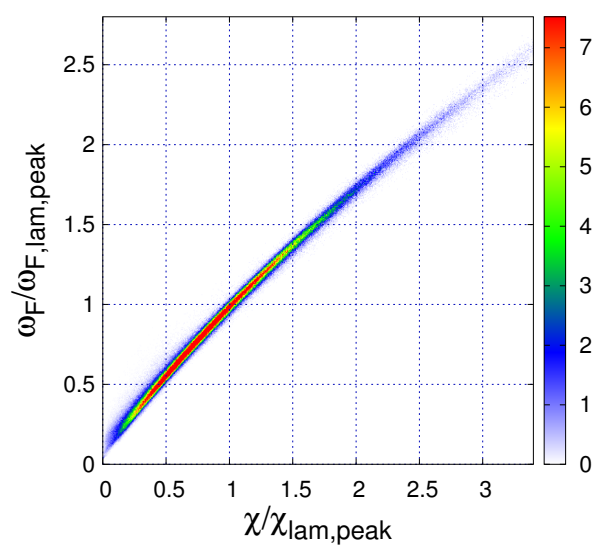

Fig. 4. Joint PDF of the fuel burning rate and the dissipation rate of the progress variable, normalized by their laminar values, on the isosurface of $c=c_{\text {peak }}$ in flame $\mathrm{B}_{n \mathrm{C} 7,1}$. 


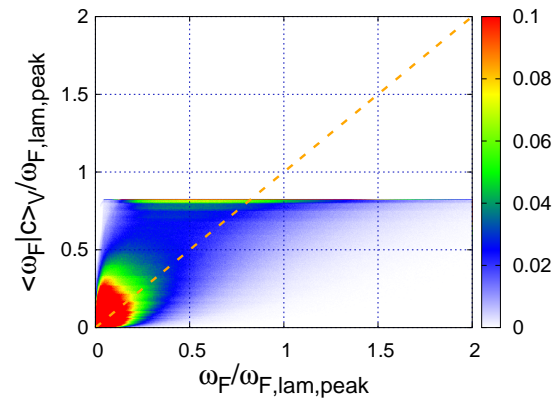

(a) $c$ alone

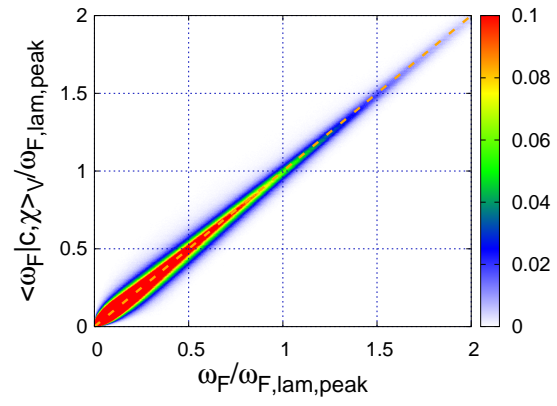

(b) $c$ and $\chi$

Fig. 5. Joint PDF of the predicted fuel burning rate and the actual burning rate in flame $\mathrm{B}_{n \mathrm{C} 7,1}$. 


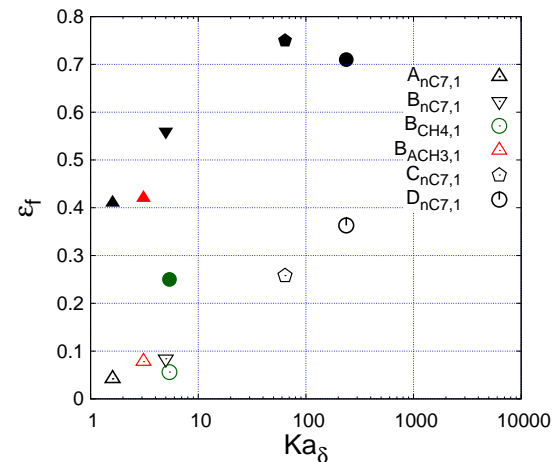

Fig. 6. Prediction error for $\left\langle\dot{\omega}_{F} \mid c\right\rangle_{V}$ (filled symbols) and $\left\langle\dot{\omega}_{F} \mid c, \chi\right\rangle_{V}$ (open symbols) as a function of the Karlovitz number at the reaction zone for all the unity Lewis number flames. 
Fig. 7. Mean of the terms in Eq 9 conditioned on $c$ for flame $\mathrm{B}_{n \mathrm{C} 7},<$ term $\mid c>$. The bars correspond to the standard deviation of these terms conditioned on $c$, $<(\text { term }-<\operatorname{term} \mid c>)^{2} \mid c>^{1 / 2}$. The red dashed line corresponds to the residual of the respective equations (Eq. A.7 in Appendix A). The labels Conv, Diff, Src, Diff-Conv, Vel cor, and Res are used for the convection, diffusion, chemical source, differential diffusion-induced convection, velocity correction, and residual terms respectively. For clarity, a description of all the terms is provided in Appendix A. Each term is normalized by the absolute value of the largest term (chemical source term) in the corresponding one-dimensional unstretched laminar flame. Note that the conditional means are evaluated as in Lapointe et al. [8]. 


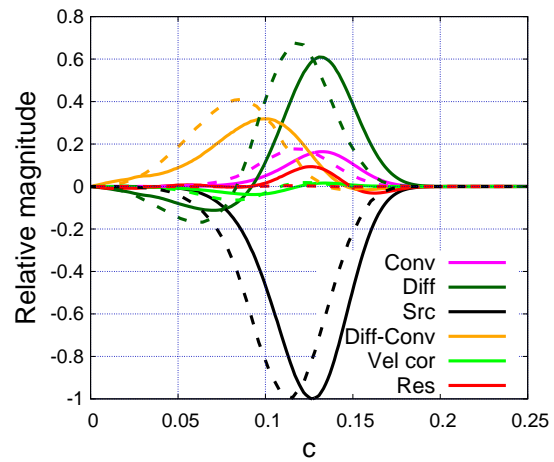

Fig. 8. Mean profiles from Fig. 7 for the ${ }_{n}-\mathrm{C}_{7} \mathrm{H}_{16}$ flamelet equation normalized to unity in both the laminar and turbulent cases. The dashed and solid lines correspond to the laminar and turbulent flames, respectively. 


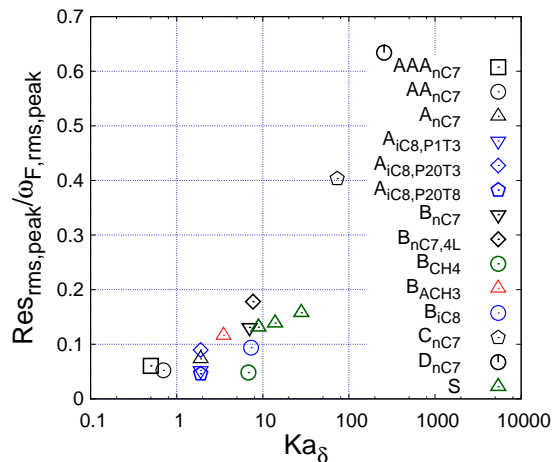

Fig. 9. Ratio of the conditional RMS of the residuals from the flamelet equation (fuel equation) to the conditional RMS of the fuel burning rate at $c=c_{\text {peak }}$ as a function of the Karlovitz number at the reaction zone for the non-unity Lewis number flames. Dashed lines are included to guide the reader with respect to the discussion in the main text. 


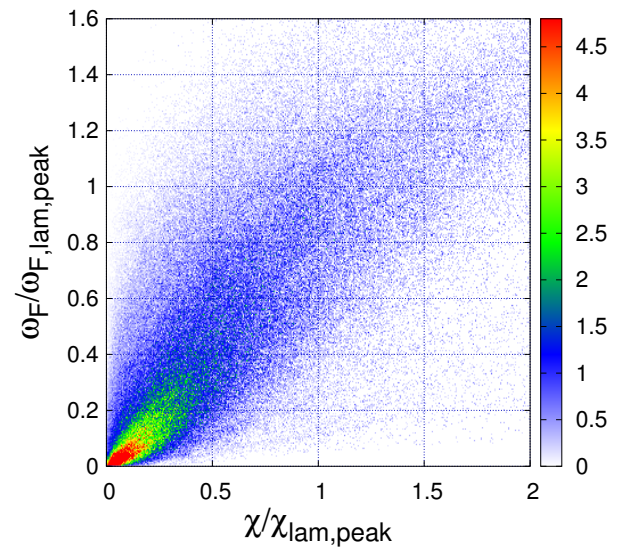

Fig. 10. Joint PDF of fuel burning rate and dissipation rate of the progress variable, normalized by their laminar values (at $c=c_{\text {lam,peak }}$ ), on the isosurface of $c=c_{\text {peak }}$ in flame $\mathrm{B}_{n \mathrm{C} 7}$. 


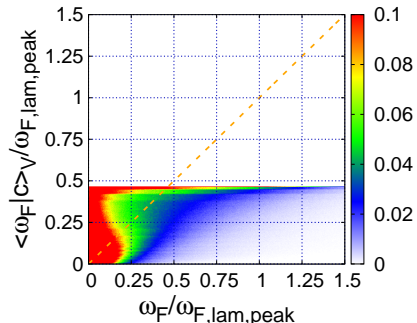

(a) c alone

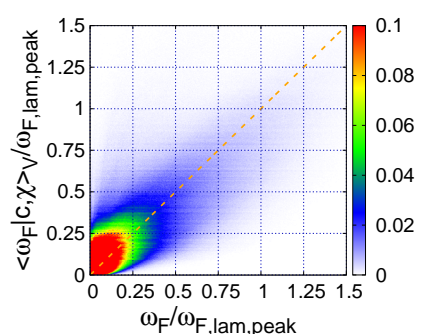

(b) $c$ and $\chi$

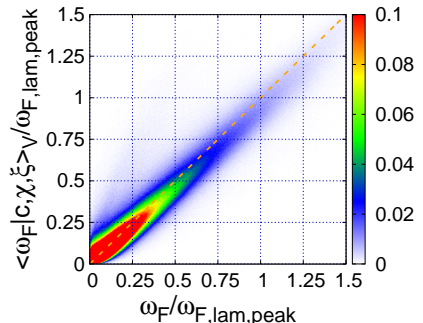

(c) $c, \chi$, and $\xi$

Fig. 11. Joint PDF of the predicted fuel burning rate and the actual burning rate in flame $\mathrm{B}_{n \mathrm{C} 7}$. 


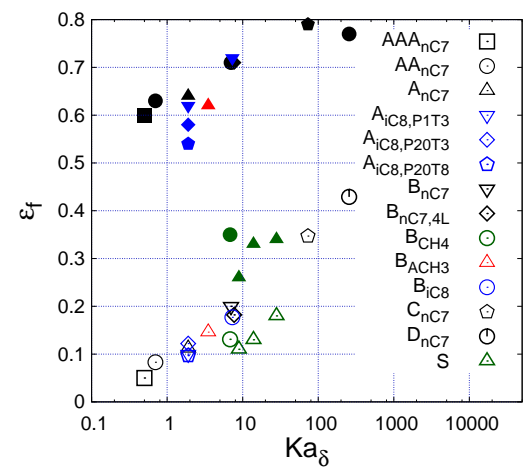

Fig. 12. Prediction error for $\left\langle\dot{\omega}_{F} \mid c\right\rangle_{V}$ (filled symbols) and $<\dot{\omega}_{F} \mid c, \chi, \xi>_{V}$ (open symbols) as a function of the Karlovitz number at the reaction zone for all the non-unity Lewis number flames. 


\section{List of Tables}

1 Simulation parameters for the set of statistically planar $n$-heptane/air flames. $\phi$ is the equivalence ratio, $T_{u}$ the unburnt temperature, $P_{0}$ the background pressure, $L e_{F}$ the Lewis number of the fuel, $S_{L}$ the laminar flame speed, $l_{F}$ the laminar flame thickness, $u^{\prime}$ the RMS of the velocity fluctuations, and $l$ the integral length scale of turbulence. $R e_{t, u}=u^{\prime} l / \nu_{u}$ is the turbulent Reynolds number in the unburnt gas, $K a_{u}=\left(l_{F} / S_{L}\right)\left(\nu_{u} l / u^{\prime 3}\right)^{-1 / 2}$ is the Karlovitz number in the unburnt gas, and $K a_{\delta}=\delta^{2} / \eta_{\delta}^{2}$ is the Karlovitz number at the reaction zone, with $\nu_{u}$ the kinematic viscosity in the unburnt gas, $\eta_{\delta}$ the Kolmogorov length scale at the reaction zone, and $\delta$ the laminar reaction zone thickness (width at $10 \%$ of peak burning rate). The laminar flame quantities are obtained with FlameMaster [51].

2 Simulation parameters for the set of statistically planar iso-octane/air, methane/air, and toluene/air flames. The parameters are defined in the caption of Table 1.

3 Simulation parameters for the slot burner flame of Sankaran et al. [39]. The parameters are defined in the caption of Table $1 . \quad 52$ 
4 Pearson's correlation coefficient [52], $r$, and distance correlation [53], dCor ${ }_{n}$, between strain rate and fuel consumption rate, curvature and fuel consumption rate, and dissipation rate and fuel consumption rate at $c=c_{\text {peak }}$ for $\mathrm{B}_{n \mathrm{C} 7,1}$.

5 Prediction error (Eq. 13) for various estimators of the fuel burning rate in the unity Lewis number $n$-heptane/air flames. $\quad 54$

6 Prediction error (Eq. 13) for various estimators of the fuel burning rate in the unity Lewis number B flames.

7 Pearson's correlation coefficient, $r$, and distance correlation, $\mathrm{dCor}_{n}$, between strain rate and fuel consumption rate, curvature and fuel consumption rate, and dissipation rate and fuel consumption rate at $c=c_{\text {peak }}$ for flame $\mathrm{B}_{n \mathrm{C} 7}$.

8 Prediction error (Eq. 13) for various estimators of the fuel burning rate in the non-unity Lewis number $n$-heptane/air, iso-octane/air, and toluene/air flames.

9 Prediction error (Eq. 13) for various estimators of the fuel burning rate in the non-unity Lewis number methane/air flames. 


\begin{tabular}{|c|c|c|c|c|c|c|c|c|c|c|c|}
\hline Case & $\mathrm{AAA}_{n \mathrm{C} 7}$ & $\mathrm{AA}_{n \mathrm{C} 7}$ & $\mathrm{~A}_{n \mathrm{C} 7}[8]$ & $\mathrm{A}_{n C 7,1}[8]$ & $\mathrm{B}_{n \mathrm{C} 7}[6]$ & $\mathrm{B}_{n C 7,4 L}[45]$ & $\mathrm{B}_{n \mathrm{C} 7,1}[6]$ & $\mathrm{C}_{n \mathrm{C} 7}[8]$ & $\mathrm{C}_{n \mathrm{C}, 1}[8]$ & $\mathrm{D}_{n \mathrm{C} 7}[8]$ & $\mathrm{D}_{n \mathrm{C} 7,1}[8]$ \\
\hline Fuel & $n-\mathrm{C}_{7} \mathrm{H}_{16}$ & $n-\mathrm{C}_{7} \mathrm{H}_{16}$ & \multirow{2}{*}{\multicolumn{2}{|c|}{$\begin{array}{c}n-\mathrm{C}_{7} \mathrm{H}_{16} \\
0.9\end{array}$}} & \multicolumn{3}{|c|}{$n-\mathrm{C}_{7} \mathrm{H}_{16}$} & \multicolumn{2}{|c|}{$n-\mathrm{C}_{7} \mathrm{H}_{16}$} & \multicolumn{2}{|c|}{$n$ - $\mathrm{C}_{7} \mathrm{H}_{16}$} \\
\hline$\phi$ & 0.9 & 0.9 & & & \multicolumn{3}{|c|}{0.9} & \multicolumn{2}{|c|}{0.9} & \multicolumn{2}{|c|}{0.9} \\
\hline$T_{u}(\mathrm{~K})$ & 298 & 298 & \multicolumn{2}{|c|}{298} & \multirow{2}{*}{\multicolumn{3}{|c|}{298}} & \multicolumn{2}{|c|}{800} & \multicolumn{2}{|c|}{800} \\
\hline$P_{0}(\mathrm{MPa})$ & 0.1 & 0.1 & \multicolumn{2}{|c|}{0.1} & & & & \multicolumn{2}{|c|}{0.1} & \multicolumn{2}{|c|}{0.1} \\
\hline$L e_{F}$ & 2.84 & 2.84 & 2.84 & 1 & & 2.84 & 1 & 2.84 & 1 & 2.84 & 1 \\
\hline$S_{L}(\mathrm{~m} / \mathrm{s})$ & 0.36 & 0.36 & 0.36 & 0.29 & \multicolumn{2}{|r|}{0.36} & 0.29 & 2.3 & 1.71 & 2.3 & 1.71 \\
\hline$l_{F}(\mathrm{~mm})$ & 0.39 & 0.39 & 0.39 & 0.43 & \multicolumn{2}{|r|}{0.39} & 0.43 & 0.25 & 0.27 & 0.25 & 0.27 \\
\hline$u^{\prime} / S_{L}$ & 2.3 & 4.6 & 9.0 & 10 & 18 & 30 & 21 & 19 & 25 & 45 & 60 \\
\hline$l / l_{F}$ & 1.1 & 1.1 & 1.1 & 1.0 & 1.1 & 4.0 & 1.0 & 1.2 & 1.1 & 1.2 & 1.1 \\
\hline$K a_{u}$ & 10 & 28 & 78 & 91 & 220 & 220 & 280 & 204 & 280 & 740 & 1050 \\
\hline$K a_{\delta}$ & 0.5 & 0.7 & 1.9 & 1.6 & 7.0 & 7.7 & 5.0 & 73 & 64 & 256 & 237 \\
\hline$R e_{t, u}$ & 24 & 49 & \multicolumn{2}{|c|}{83} & 190 & 1150 & 190 & \multicolumn{2}{|c|}{170} & \multicolumn{2}{|c|}{380} \\
\hline
\end{tabular}

Table 1

Simulation parameters for the set of statistically planar $n$-heptane/air flames. $\phi$ is the equivalence ratio, $T_{u}$ the unburnt temperature, $P_{0}$ the background pressure, $L e_{F}$ the Lewis number of the fuel, $S_{L}$ the laminar flame speed, $l_{F}$ the laminar flame thickness, $u^{\prime}$ the RMS of the velocity fluctuations, and $l$ the integral length scale of turbulence. $R e_{t, u}=u^{\prime} l / \nu_{u}$ is the turbulent Reynolds number in the unburnt gas, $K a_{u}=\left(l_{F} / S_{L}\right)\left(\nu_{u} l / u^{\prime 3}\right)^{-1 / 2}$ is the Karlovitz number in the unburnt gas, and $K a_{\delta}=\delta^{2} / \eta_{\delta}^{2}$ is the Karlovitz number at the reaction zone, with $\nu_{u}$ the kinematic viscosity in the unburnt gas, $\eta_{\delta}$ the Kolmogorov length scale at the reaction zone, and $\delta$ the laminar reaction zone thickness (width at $10 \%$ of peak burning rate). The laminar flame quantities are obtained with FlameMaster [51]. 


\begin{tabular}{|c|c|c|c|c|c|c|c|c|}
\hline Case & $\mathrm{A}_{i \mathrm{C} 8, \mathrm{P} 1 \mathrm{~T} 3}[10]$ & $\mathrm{A}_{i \mathrm{C} 8, \mathrm{P} 20 \mathrm{~T} 3}[10]$ & $\mathrm{A}_{i \mathrm{C} 8, \mathrm{P} 20 \mathrm{~T} 8}[10]$ & $\mathrm{B}_{\mathrm{CH} 4}[9]$ & $\mathrm{B}_{\mathrm{CH} 4,1}[9]$ & $\mathrm{B}_{\mathrm{ACH} 3}[9]$ & $\mathrm{B}_{\mathrm{ACH} 3,1}[9]$ & $\mathrm{B}_{i \mathrm{C} 8}[10]$ \\
\hline Fuel & $i-\mathrm{C}_{8} \mathrm{H}_{18}$ & $i-\mathrm{C}_{8} \mathrm{H}_{18}$ & $i-\mathrm{C}_{8} \mathrm{H}_{18}$ & \multicolumn{2}{|c|}{$\mathrm{CH}_{4}$} & \multicolumn{2}{|c|}{$\mathrm{A}_{1}-\mathrm{CH}_{3}$} & $i-\mathrm{C}_{8} \mathrm{H}_{18}$ \\
\hline$\phi$ & 0.9 & 0.9 & 0.9 & \multicolumn{2}{|c|}{0.9} & \multicolumn{2}{|c|}{0.9} & 0.9 \\
\hline$T_{u}(\mathrm{~K})$ & 298 & 298 & 800 & \multicolumn{2}{|c|}{298} & \multicolumn{2}{|c|}{298} & 298 \\
\hline$P_{0}(\mathrm{MPa})$ & 0.1 & 2 & 2 & \multicolumn{2}{|c|}{0.1} & \multicolumn{2}{|c|}{0.1} & 0.1 \\
\hline$L e_{F}$ & 2.49 & 2.49 & 2.49 & 0.99 & 1 & 2.45 & 1 & 2.49 \\
\hline$S_{L}(\mathrm{~m} / \mathrm{s})$ & 0.32 & 0.11 & 0.93 & 0.33 & 0.26 & 0.35 & 0.27 & 0.32 \\
\hline$l_{F}(\mathrm{~mm})$ & 0.40 & 0.045 & 0.021 & 0.47 & 0.41 & 0.41 & 0.49 & 0.40 \\
\hline$u^{\prime} / S_{L}$ & 7.6 & 20 & 3.7 & 16 & 20 & 17 & 22 & 19 \\
\hline$l / l_{F}$ & 1.0 & 1.0 & 1.0 & 1.1 & 0.9 & 1.1 & 0.9 & 1.0 \\
\hline$K a_{u}$ & 61 & 234 & 16 & 190 & 270 & 205 & 324 & 234 \\
\hline$K a_{\delta}$ & 1.9 & 1.9 & 1.9 & 6.8 & 5.4 & 3.5 & 3.1 & 7.3 \\
\hline$R e_{t, u}$ & 65 & 140 & 18 & \multicolumn{2}{|c|}{160} & \multicolumn{2}{|c|}{175} & 159 \\
\hline
\end{tabular}

Table 2

Simulation parameters for the set of statistically planar iso-octane/air, methane/air, and toluene/air flames. The parameters are defined in the caption of Table 1. 


\begin{tabular}{l|c|c|c}
\hline Case & $\mathrm{S}^{1 / 4}$ & $\mathrm{~S}^{1 / 2}$ & $\mathrm{~S}^{3 / 4}$ \\
\hline Fuel & $\mathrm{CH}_{4}$ & $\mathrm{CH}_{4}$ & $\mathrm{CH}_{4}$ \\
$\phi$ & 0.7 & 0.7 & 0.7 \\
$T_{u}(\mathrm{~K})$ & 800 & 800 & 800 \\
$P_{0}(\mathrm{MPa})$ & 0.1 & 0.1 & 0.1 \\
$L e_{F}$ & 0.96 & 0.96 & 0.96 \\
$S_{L}(\mathrm{~m} / \mathrm{s})$ & 1.8 & 1.8 & 1.8 \\
$l_{F}(\mathrm{~mm})$ & 0.32 & 0.32 & 0.32 \\
$u^{\prime} / S_{L}$ & 9.8 & 5.5 & 4.0 \\
$l / l_{F}$ & 2.2 & 2.5 & 3.9 \\
$K a_{u}$ & 50 & 20 & 10 \\
$K a_{\delta}$ & 28 & 13.9 & 8.9 \\
$R e_{t, u}$ & 143 & 92.4 & 106 \\
\hline
\end{tabular}

Table 3

Simulation parameters for the slot burner flame of Sankaran et al. [39]. The parameters are defined in the caption of Table 1. 


\begin{tabular}{lcc}
\hline & $r$ & $\mathrm{dCor}_{n}$ \\
\hline Strain rate & 0.61 & 0.57 \\
Curvature & -0.12 & 0.33 \\
Dissipation rate & 0.996 & 0.997 \\
\hline
\end{tabular}

Table 4

Pearson's correlation coefficient [52], $r$, and distance correlation [53], dCor ${ }_{n}$, between strain rate and fuel consumption rate, curvature and fuel consumption rate, and dissipation rate and fuel consumption rate at $c=c_{\text {peak }}$ for $\mathrm{B}_{n \mathrm{C} 7,1}$. 


\begin{tabular}{lcccc}
\hline Estimator $f$ & $\mathrm{~A}_{n \mathrm{C} 7,1}$ & $\mathrm{~B}_{n \mathrm{C} 7,1}$ & $\mathrm{C}_{n \mathrm{C} 7,1}$ & $\mathrm{D}_{n \mathrm{C} 7,1}$ \\
\hline$<\dot{\omega}_{F} \mid c>_{V}$ & 0.41 & 0.56 & 0.75 & 0.71 \\
$<\dot{\omega}_{F} \mid c, \kappa>_{V}$ & 0.34 & 0.49 & 0.72 & 0.70 \\
$<\dot{\omega}_{F} \mid c, a_{t}>_{V}$ & 0.32 & 0.46 & 0.71 & 0.70 \\
$<\dot{\omega}_{F} \mid c, \chi>_{V}$ & 0.04 & 0.08 & 0.26 & 0.36 \\
\hline
\end{tabular}

Table 5

Prediction error (Eq. 13) for various estimators of the fuel burning rate in the unity Lewis number $n$-heptane/air flames. 
Table 6

\begin{tabular}{lccc}
\hline Estimator $f$ & $\mathrm{~B}_{n \mathrm{C} 7,1}$ & $\mathrm{~B}_{\mathrm{CH} 4,1}$ & $\mathrm{~B}_{\mathrm{ACH} 3,1}$ \\
\hline$<\dot{\omega}_{F} \mid c>_{V}$ & 0.56 & 0.25 & 0.42 \\
$<\dot{\omega}_{F} \mid c, \kappa>_{V}$ & 0.49 & 0.21 & 0.36 \\
$<\dot{\omega}_{F} \mid c, a_{t}>_{V}$ & 0.46 & 0.21 & 0.35 \\
$<\dot{\omega}_{F} \mid c, \chi>_{V}$ & 0.08 & 0.06 & 0.08 \\
\hline
\end{tabular}

Prediction error (Eq. 13) for various estimators of the fuel burning rate in the unity Lewis number B flames. 


\begin{tabular}{lcc}
\hline & $r$ & $\mathrm{dCor}_{n}$ \\
\hline Strain rate & 0.49 & 0.45 \\
Curvature & -0.53 & 0.65 \\
Dissipation rate & 0.75 & 0.72 \\
\hline
\end{tabular}

Table 7

Pearson's correlation coefficient, $r$, and distance correlation, $\mathrm{dCor}_{n}$, between strain rate and fuel consumption rate, curvature and fuel consumption rate, and dissipation rate and fuel consumption rate at $c=c_{\text {peak }}$ for flame $\mathrm{B}_{n \mathrm{C} 7}$. 


\begin{tabular}{|c|c|c|c|c|}
\hline Estimator $f$ & \multicolumn{3}{|c|}{ 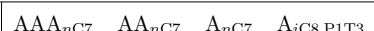 } & $\mathrm{A}_{i \mathrm{C} 8 \mathrm{P} 20 \mathrm{~T} 3}$ \\
\hline
\end{tabular}

\begin{tabular}{l|llllllllllll}
$\left\langle\dot{\omega}_{F} \mid c, \chi\right\rangle_{V}$ & 0.15 & 0.27 & 0.34 & 0.32 & 0.34 & 0.32 & 0.43 & 0.40 & 0.36 & 0.41 & 0.55 & 0.58
\end{tabular}

\begin{tabular}{l|llllllllllll}
$<\dot{\omega}_{F} \mid c, \chi, \kappa>_{V}$ & 0.11 & 0.17 & 0.21 & 0.18 & 0.20 & 0.17 & 0.31 & 0.32 & 0.26 & 0.31 & 0.50 & 0.54
\end{tabular}

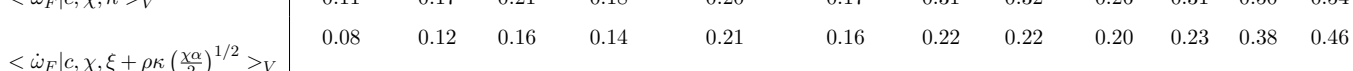

$<\dot{\omega}_{F} \mid c, \chi, \xi>_{V}$

$\begin{array}{lllll}0.05 & 0.08 & 0.11 & 0.10 & 0.12\end{array}$

$\begin{array}{lllllll}0.10 & 0.20 & 0.18 & 0.15 & 0.18 & 0.35 & 0.43\end{array}$

Table 8

Prediction error (Eq. 13) for various estimators of the fuel burning rate in the nonunity Lewis number $n$-heptane/air, iso-octane/air, and toluene/air flames. 


\begin{tabular}{l|cccc}
\hline Estimator $f$ & $\mathrm{~B}_{\mathrm{CH} 4}$ & $\mathrm{~S}^{1 / 4}$ & $\mathrm{~S}^{1 / 2}$ & $\mathrm{~S}^{3 / 4}$ \\
\hline$<\dot{\omega}_{F} \mid c>_{V}$ & 0.35 & 0.34 & 0.33 & 0.26 \\
$<\dot{\omega}_{F} \mid c, \chi>_{V}$ & 0.22 & 0.25 & 0.21 & 0.18 \\
$<\dot{\omega}_{F} \mid c, \chi, \kappa>_{V}$ & 0.15 & 0.21 & 0.15 & 0.11 \\
& 0.18 & 0.20 & 0.17 & 0.16 \\
$<\dot{\omega}_{F} \mid c, \chi, \xi+\rho \kappa\left(\frac{\chi \alpha}{2}\right)^{1 / 2}>_{V}$ & & & & \\
$<\dot{\omega}_{F} \mid c, \chi, \xi>_{V}$ & 0.13 & 0.18 & 0.13 & 0.11 \\
\hline
\end{tabular}

Table 9

Prediction error (Eq. 13) for various estimators of the fuel burning rate in the nonunity Lewis number methane/air flames. 